\title{
Investigation of internal efforts in the components of the crop sprayer boom section
}

\author{
R. Hevko a, M. Stashkiv a, O. Lyashuk b, Y. Vovk b,*, \\ V. Oleksyuk a, O. Tson b, I. Bortnyk a \\ a Department of Technical Mechanics and Agricultural Machinery, Ternopil Ivan Puluj \\ National Technical University, 56 Ruska str., Ternopil 46001, Ukraine \\ b Department of Automobiles, Ternopil Ivan Puluj National Technical University, \\ 56 Ruska str., Ternopil 46001, Ukraine \\ * Corresponding e-mail address: vovkyuriy@ukr.net \\ ORCID identifier: (1) https://orcid.org/0000-0001-7569-2943 (R.H.); (1) https://orcid.org/0000- \\ 0002-7325-8016 (M.S.); (1) https://orcid.org/0000-0003-4881-8568 (O.L.); (1)https://orcid. \\ org/0000-0001-8983-2580 (Y.V.); (1) https://orcid.org/0000-0003-2795-590X (V.O.); Dhttps:// \\ orcid.org/0000-0003-1056-4697 (O.T.); (1) https://orcid.org/0000-0003-0982-4826 (I.B.)
}

\section{ABSTRAGT}

Purpose: Development results allow the defects in the boom section of a crop spraying machine design to be found, the most loaded structural components to be determined and the most suitable design parameters to be chosen.

Design/methodology/approach: Results of theoretical investigation of internal forces distribution in the components of a boom section of a crop spraying machine in the static statement of the problem have been presented. The applied under discussion enables the static indeterminacy to be realized whilst calculating the complex frame structures though it requires not only considerable records of the function of potential energy of deformation (even without taking into account the function of potential energy of deformation of normal and lateral forces) but application of applied computer programs. The technique of static indeterminacy realization by minimum of potential energy of deformation (MPED) method has been used to calculate the framework.

Findings: Technique of static indeterminacy realization by minimum of potential energy of deformation (MPED) method has been used to calculate the framework. The results of theoretical calculation and simulation of the boom section of a crop spraying machine have been compared. The results of analytical calculation have been checked by the simulation of 3D model of the using the program complex «Lira-SAPR», where the finite elements method (FEM) has been implemented.

Research limitations/implications: Horizontal components of the shear forces as well as the normal forces and as a consequence the corresponding potential deformation energy are neglected, which has some effect on the accuracy of the calculations.

Practical implications: An effective tool for strength analysis with preliminary assessment and diagnostics structures based on the constructed calculation models of stress strain state load-bearing frames of typical geometry with an arbitrarily given distribution of external load. 
Originality/value: A universal algorithm records of the function of torque and bending moment, as well as the function of potential deformation energy of a spraying machine boom section.

Keywords: Potential energy of deformation, Static indeterminacy, Loadbearing structure, Boom section of a crop spraying machine, Simulation

\section{Reference to this paper should be given in the following way:}

R. Hevko, M. Stashkiv, O. Lyashuk, Y. Vovk, V. Oleksyuk, O. Tson, I. Bortnyk, Investigation of internal efforts in the components of the crop sprayer boom section, Journal of Achievements in Materials and Manufacturing Engineering 105/1 (2021) 33-41. DOI: https://doi.org/10.5604/01.3001.0014.8743

\section{ANALYSIS AND MODELLING}

\section{Introduction}

Protection of plants against diseases and crop pests is the necessary process in the technology of agricultural crops production and it is especially important under intensive agricultural production conditions. At present protective measures can be taken by different methods: agro technical, physical-mechanical, biological, chemical and others. The chemical method involves approximately $90 \%$ of ways of crop protection against diseases and crop pests. The method is based on the use of fungicidal agents, organic and nonorganic mixtures, which are toxic for phytopathogenes. Such method of crops protection has been implemented by means of special equipment - spraying machines.

Nowadays, there are a great number of design options of crop sprayers which are produced by different companies. They vary in design, performance and technological parameters (capacity, band width, quality of technological operation performed and others). The necessity of using of any of these machines to apply crop protection agents is specified in each certain case taking into account different application conditions, namely technological (fertilizer application rate, crop growth phase), climate (air temperature, wind velocity), geographical (soil relief) and relevant environmental aspects [1].

The increased requirements to the loadbearing systems of machines for chemical protection of crops referred to the fact that they usually operate under difficult performance and relief-climate conditions. The most frequent reasons of machines breakdown are design errors (20-30\%), machine failure due to its low quality of manufacture and assembly (20-30\%), low technical level and quality of materials and accessories (35-40\%), failures due to the violations of rules of machines maintenance in the companies and low qualifications of staff $(10 \%)$, other failures $-5-10 \%[2,3]$.

One of the main reasons of failures of machines for crops chemical protection, namely, the crop sprayer of wide band
(25 $\mathrm{m}$ and more) boom section frames in particular, is the loss of bearing capacity, caused by the design imperfection and insufficient strength margin of its components $[4,5]$.

To calculate the mobile machines frameworks a great number of methods which are different in complexity and accuracy of obtained results have been developed which take onto account the specific features of their design. The most typical among them are random space pattern, use of components of different types, complex and unpredictable loading conditions [6-10]. Moreover, the process of calculation of machines frameworks is considerably complicated by the high degree of their static indeterminacy. In this case whilst developing the theoretical principles of "excessive" binding removal of machines frameworks the method based on the principle of minimum of potential energy of deformation has appeared to be quite efficient [5].

Potential energy of deformation is a homogeneous function of the second degree of the external forces or displacement [11]:

$$
\begin{aligned}
& U=\sum \int \frac{1}{2 E A} \cdot N^{2} d s+\sum \int \frac{1}{2 k G A} \cdot Q^{2} d s+\sum \int \frac{1}{2 E I} \cdot M^{2} d s+ \\
& \sum \int \frac{1}{2 G I_{P}} \cdot T^{2} d s
\end{aligned}
$$

where $E$ and $G$ - elasticity modulus of the first and second kinds respectively, $\mathrm{MPa} ; A$ - area of the component cross section, $\mathrm{m}^{2} ; I, I_{P}$ - the component cross section axial and polar moment of inertia respectively, $\mathrm{m}^{4} ; N, Q$-longitudinal and lateral forces respectively, $\mathrm{N}$ (about the longitudinal axis of the component); $M, T$ - bending and torque moments respectively, $\mathrm{N} \mathrm{m} ; s$ - the way along which the integration takes place, $\mathrm{m}$.

According to the Castigliano theorem, the partial derivative of the potential energy equation by the required internal efforts results in displacement equal to this effort. If "excessive" bindings are such that the displacement by the direction of their unknown reactions $X_{i}$ is equal to zero, so, according to the theorem of the smallest work: 


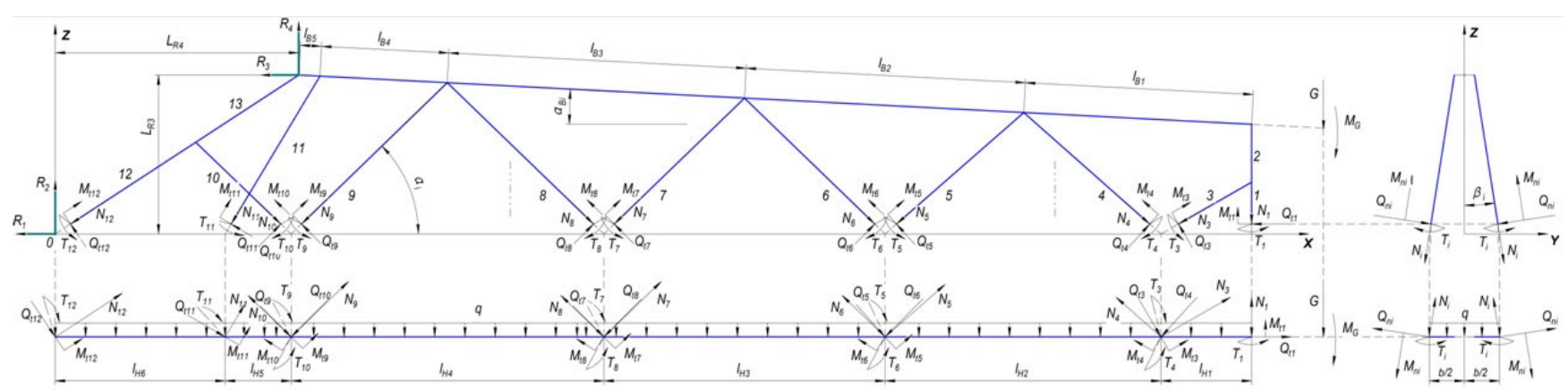

Fig. 1. Design model of crop sprayer boom section

$\frac{\partial U}{\partial X_{1}}=0$

$\frac{\partial U}{\partial X_{2}}=0$

$\frac{\partial U}{\partial X_{n}}=0$

that allows the necessary additional equations to determine the unknown internal efforts to be obtained.

Using such method of static indeterminacy realization of complex multicomponent structures, the approach when the expression of function of potential energy of deformation is written only for the constituents corresponding to the bending deformation and torsion is also a reasonable one.

$U=\sum \int \frac{1}{2 E I} \cdot M^{2} d s+\sum \int \frac{1}{2 G I_{P}} \cdot T^{2} d s ;$

$\frac{\partial U}{\partial M_{i}}=0$

$\frac{\partial U}{\partial T_{i}}=0$

The above-mentioned approach has greatly reduced the expression length of function of potential energy of deformation and the number of mathematical operations to determine the unknown internal efforts under accessible decrease in engineering calculation accuracy conditions [12-16].

\section{Methodology}

Due to the above-mentioned method the calculation of the crop sprayer boom section has been performed. Whilst solving the problem the implemented design model is described in Figure 1.

The following assumptions have been taken at design model development:

1. We take into consideration the internal efforts only in the members of the first section of the boom, the impact of middle and end sections is simulated by the action of external force $G$ and moment $M_{G}$.

2. Loading $q$ of the processing equipment is considered to be uniformly distributed and applied to the lower spar of the boom.

3. To make the recording of function of potential energy of deformation more convenient we have cut the boom into two parts (Fig. 1): into two symmetrical parts by vertical cutting plane on the boom line of symmetry and horizontal cutting plane above the boom lower spar.

The interdependence of cut members has been simulated in couples which are the same in size but different in direction of internal efforts: $N$ - the force, acting along the component axis; $Q_{n}$ and $Q_{\tau}$ - projections of lateral force on the element in normal and tangential planes (about the boom longitudinal axis); $M_{n}, M_{\tau}$ and $T_{n}, T_{\tau}$-bending and torque moments projections respectively; $\alpha_{i}$ - angle of inclination of $i$ structure component to horizontal plane; $\beta_{i}$ - angle of inclination of $i$ structure component to vertical plane; $l, l_{B}, l_{H}$ - lengths of tilt brace and components of upper and lower spars respectively.

In Figure 2 shows a design model of crop sprayer boom section enlarged and divided into two parts (right to left) for a better view.

The crop sprayer boom section is made of thin-walled elements of different cross-section. The general crosssections view of the upper spar, lower spar and tilt brace are shown in the Figure 3.

The geometrical characteristics of the thin-walled elements cross - sections are: for the upper spar $A=$ $8.64 \mathrm{~cm}^{2}, I_{x}=32.17 \mathrm{~cm}^{4} ; I_{y}=81.96 \mathrm{~cm}^{4}, I_{p}=0.49 \mathrm{~cm}^{4}$; for the lower spar $A=12.54 \mathrm{~cm}^{2}, I_{x}=38.04 \mathrm{~cm}^{4} ; I_{y}=1300 \mathrm{~cm}^{4}$, $I_{p}=0.48 \mathrm{~cm}^{4}$; for the tilt brace $A=2.24 \mathrm{~cm}^{2}, I_{x}=I_{y}=$ $2.94 \mathrm{~cm}^{4}, I_{p}=4.56 \mathrm{~cm}^{4}$. 


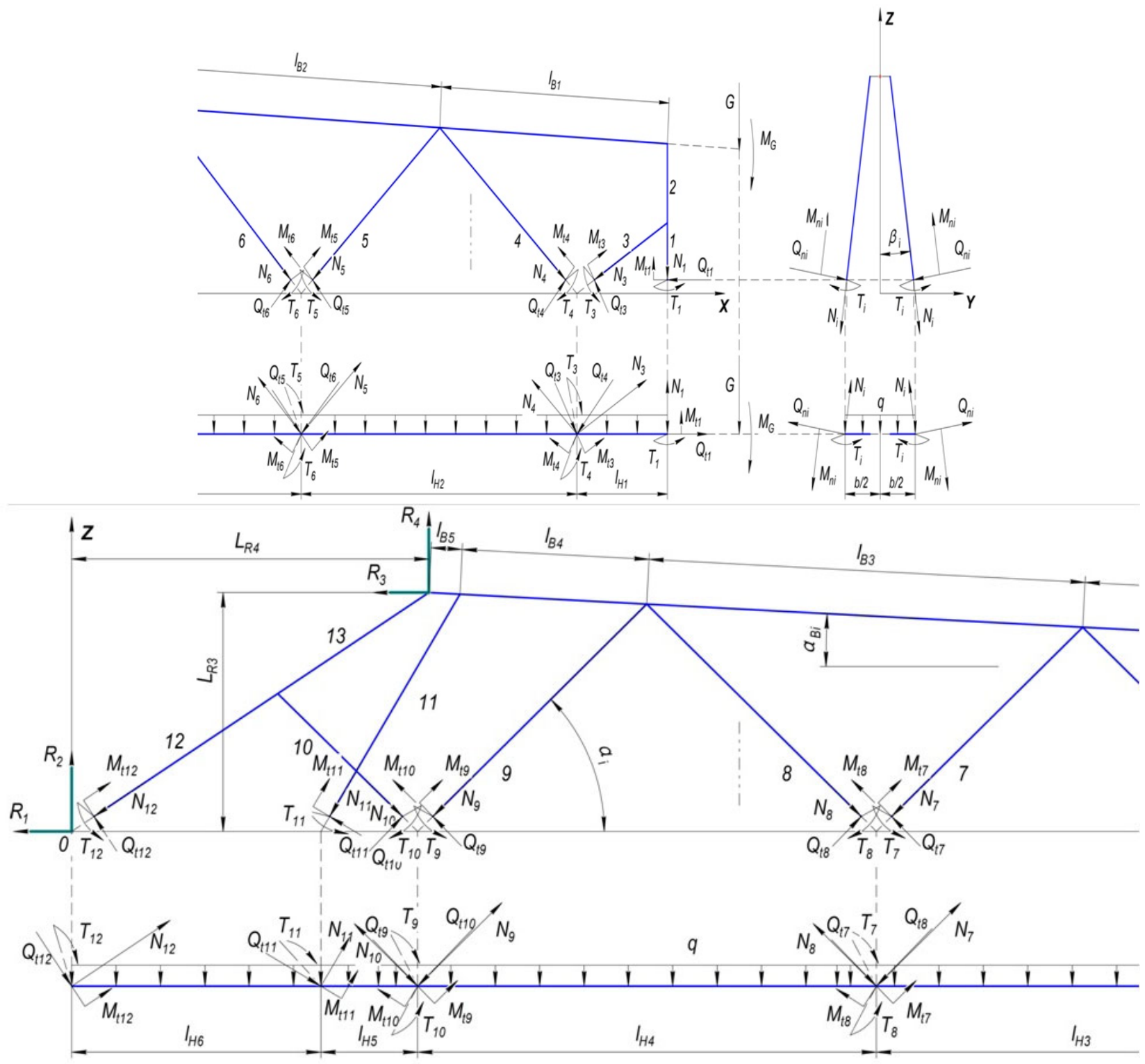

Fig. 2. Enlarged design model of crop sprayer boom section

a)

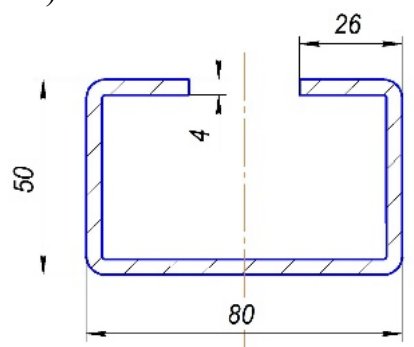

b)

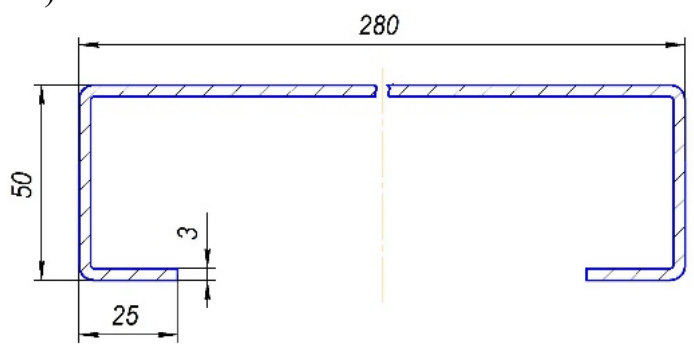

c)

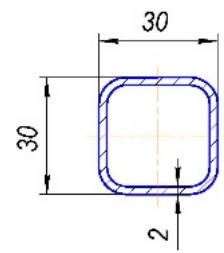

Fig. 3. Cross-sections of crop sprayer boom section elements: a) upper spar; b) lower spar; c) tilt brace 
As an example, we will write the expression of the function of potential energy of deformation of the bending and torque moments (only for the lower spar of the boom section).

The function of potential energy of deformation of the bending moments:

$U_{\text {Bend }}\left(l_{H 2}\right)=\frac{1}{2 E I} \int_{0}^{l_{H 2}}\left(M_{G}+G \cdot\left(x+l_{H 1}\right)+q \frac{\left(x+l_{H 1}\right)^{2}}{2}-N_{1} \cdot \cos \beta_{1} \cdot\left(x+l_{H 1}\right)-Q_{n 1} \cdot \sin \beta_{1} \cdot\left(x+l_{H 1}\right)-N_{3} \cdot \sin \alpha_{3}\right.$. $\cos \beta_{3} \cdot x-Q_{n 3} \cdot \sin \beta_{3} \cdot x+Q_{\tau 3} \cdot \cos \alpha_{3} \cdot \cos \beta_{3} \cdot x-N_{4} \cdot \sin \alpha_{4} \cdot \cos \beta_{4} \cdot x-Q_{n 4} \cdot \sin \beta_{4} \cdot x+Q_{\tau 4} \cdot \cos \alpha_{4} \cdot \cos \beta_{4} \cdot$ $\left.x-M_{\tau 3} \cdot \cos \beta_{3}+M_{\tau 4} \cdot \cos \beta_{4}+T_{3} \cdot \sin \beta_{3}-T_{4} \cdot \sin \beta_{4}\right)^{2} d x$;

$U_{\text {Bend }}\left(l_{H 3}\right)=\frac{1}{2 E I} \int_{0}^{l_{H 3}}\left(M_{G}+G \cdot\left(x+l_{H 1}+l_{H 2}\right)+q \frac{\left(x+l_{H 1}+l_{H 2}\right)^{2}}{2}-N_{1} \cdot \cos \beta_{1} \cdot\left(x+l_{H 1}+l_{H 2}\right)-Q_{n 1} \cdot\right.$ $\sin \beta_{1} \cdot\left(x+l_{H 1}+l_{H 2}\right)-N_{3} \cdot \sin \alpha_{3} \cdot \cos \beta_{3} \cdot\left(x+l_{H 2}\right)-Q_{n 3} \cdot \sin \beta_{3} \cdot\left(x+l_{H 2}\right)+Q_{\tau 3} \cdot \cos \alpha_{3} \cdot \cos \beta_{3} \cdot\left(x+l_{H 2}\right)-N_{4} \cdot$ $\sin \alpha_{4} \cdot \cos \beta_{4} \cdot\left(x+l_{H 2}\right)-Q_{n 4} \cdot \sin \beta_{4} \cdot\left(x+l_{H 2}\right)+Q_{\tau 4} \cdot \cos \alpha_{4} \cdot \cos \beta_{4} \cdot\left(x+l_{H 2}\right)-M_{\tau 3} \cdot \cos \beta_{3}+M_{\tau 4} \cdot \cos \beta_{4}+T_{3}$. $\sin \beta_{3}-T_{4} \cdot \sin \beta_{4}-N_{5} \cdot \sin \alpha_{5} \cdot \cos \beta_{5} \cdot x-Q_{n 5} \cdot \sin \beta_{5} \cdot x+Q_{\tau 5} \cdot \cos \alpha_{5} \cdot \cos \beta_{5} \cdot x-N_{6} \cdot \sin \alpha_{6} \cdot \cos \beta_{6} \cdot x-Q_{n 6}$. $\left.\sin \beta_{6} \cdot x+Q_{\tau 6} \cdot \cos \alpha_{6} \cdot \cos \beta_{6} \cdot x-M_{\tau 5} \cdot \cos \beta_{5}+M_{\tau 6} \cdot \cos \beta_{6}+T_{5} \cdot \sin \beta_{5}-T_{6} \cdot \sin \beta_{6}\right)^{2} d x ;$ $U_{B e n d}\left(l_{H 4}\right)=\frac{1}{2 E I} \int_{0}^{l_{H 4}}\left(M_{G}+G \cdot\left(x+l_{H 1}+l_{H 2}+l_{H 3}\right)+q \frac{\left(x+l_{H 1}+l_{H 2}+l_{H 3}\right)^{2}}{2}-N_{1} \cdot \cos \beta_{1} \cdot\left(x+l_{H 1}+l_{H 2}+l_{H 3}\right)-Q_{n 1}\right.$. $\sin \beta_{1} \cdot\left(x+l_{H 1}+l_{H 2}+l_{H 3}\right)-N_{3} \cdot \sin \alpha_{3} \cdot \cos \beta_{3} \cdot\left(x+l_{H 2}+l_{H 3}\right)-Q_{n 3} \cdot \sin \beta_{3} \cdot\left(x+l_{H 2}+l_{H 3}\right)+Q_{\tau 3} \cdot \cos \alpha_{3} \cdot$ $\cos \beta_{3} \cdot\left(x+l_{H 2}+l_{H 3}\right)-N_{4} \cdot \sin \alpha_{4} \cdot \cos \beta_{4} \cdot\left(x+l_{H 2}+l_{H 3}\right)-Q_{n 4} \cdot \sin \beta_{4} \cdot\left(x+l_{H 2}+l_{H 3}\right)+Q_{\tau 4} \cdot \cos \alpha_{4} \cdot \cos \beta_{4} \cdot$ $\left(x+l_{H 2}+l_{H 3}\right)-M_{\tau 3} \cdot \cos \beta_{3}+M_{\tau 4} \cdot \cos \beta_{4}+T_{3} \cdot \sin \beta_{3}-T_{4} \cdot \sin \beta_{4}-N_{5} \cdot \sin \alpha_{5} \cdot \cos \beta_{5} \cdot\left(x+l_{H 3}\right)-Q_{n 5} \cdot \sin \beta_{5}$. $\left(x+l_{H 3}\right)+Q_{\tau 5} \cdot \cos \alpha_{5} \cdot \cos \beta_{5} \cdot\left(x+l_{H 3}\right)-N_{6} \cdot \sin \alpha_{6} \cdot \cos \beta_{6} \cdot\left(x+l_{H 3}\right)-Q_{n 6} \cdot \sin \beta_{6} \cdot\left(x+l_{H 3}\right)+Q_{\tau 6} \cdot \cos \alpha_{6} \cdot$ $\cos \beta_{6} \cdot\left(x+l_{H 3}\right)-M_{\tau 5} \cdot \cos \beta_{5}+M_{\tau 6} \cdot \cos \beta_{6}+T_{5} \cdot \sin \beta_{5}-T_{6} \cdot \sin \beta_{6}-N_{7} \cdot \sin \alpha_{7} \cdot \cos \beta_{7} \cdot x-Q_{n 7} \cdot \sin \beta_{7} \cdot x+Q_{\tau 7} \cdot$ $\cos \alpha_{7} \cdot \cos \beta_{7} \cdot x-N_{8} \cdot \sin \alpha_{8} \cdot \cos \beta_{8} \cdot x-Q_{n 8} \cdot \sin \beta_{8} \cdot x+Q_{\tau 8} \cdot \cos \alpha_{8} \cdot \cos \beta_{8} \cdot x-M_{\tau 7} \cdot \cos \beta_{7}+M_{\tau 8} \cdot \cos \beta_{8}+T_{7}$. $\left.\sin \beta_{7}-T_{8} \cdot \sin \beta_{8}\right)^{2} d x$;

$U_{B e n d}\left(l_{H 5}\right)=\frac{1}{2 E I} \int_{0}^{l_{H 5}}\left(M_{G}+G \cdot\left(x+l_{H 1}+l_{H 2}+l_{H 3}+l_{H 4}\right)+q \frac{\left(x+l_{H 1}+l_{H 2}+l_{H 3}+l_{H 4}\right)^{2}}{2}-N_{1} \cdot \cos \beta_{1} \cdot\left(x+l_{H 1}+l_{H 2}+\right.\right.$ $\left.l_{H 3}+l_{H 4}\right)-Q_{n 1} \cdot \sin \beta_{1} \cdot\left(x+l_{H 1}+l_{H 2}+l_{H 3}+l_{H 4}\right)-N_{3} \cdot \sin \alpha_{3} \cdot \cos \beta_{3} \cdot\left(x+l_{H 2}+l_{H 3}+l_{H 4}\right)-Q_{n 3} \cdot \sin \beta_{3} \cdot$ $\left(x+l_{H 2}+l_{H 3}+l_{H 4}\right)+Q_{\tau 3} \cdot \cos \alpha_{3} \cdot \cos \beta_{3} \cdot\left(x+l_{H 2}+l_{H 3}+l_{H 4}\right)-N_{4} \cdot \sin \alpha_{4} \cdot \cos \beta_{4} \cdot\left(x+l_{H 2}+l_{H 3}+l_{H 4}\right)-Q_{n 4}$. $\sin \beta_{4} \cdot\left(x+l_{H 2}+l_{H 3}+l_{H 4}\right)+Q_{\tau 4} \cdot \cos \alpha_{4} \cdot \cos \beta_{4} \cdot\left(x+l_{H 2}+l_{H 3}+l_{H 4}\right)-M_{\tau 3} \cdot \cos \beta_{3}+M_{\tau 4} \cdot \cos \beta_{4}+T_{3} \cdot \sin \beta_{3}-$ $T_{4} \cdot \sin \beta_{4}-N_{5} \cdot \sin \alpha_{5} \cdot \cos \beta_{5} \cdot\left(x+l_{H 3}+l_{H 4}\right)-Q_{n 5} \cdot \sin \beta_{5} \cdot\left(x+l_{H 3}+l_{H 4}\right)+Q_{\tau 5} \cdot \cos \alpha_{5} \cdot \cos \beta_{5} \cdot\left(x+l_{H 3}+l_{H 4}\right)-$ $N_{6} \cdot \sin \alpha_{6} \cdot \cos \beta_{6} \cdot\left(x+l_{H 3}+l_{H 4}\right)-Q_{n 6} \cdot \sin \beta_{6} \cdot\left(x+l_{H 3}+l_{H 4}\right)+Q_{\tau 6} \cdot \cos \alpha_{6} \cdot \cos \beta_{6} \cdot\left(x+l_{H 3}+l_{H 4}\right)-M_{\tau 5} \cdot$ $\cos \beta_{5}+M_{\tau 6} \cdot \cos \beta_{6}+T_{5} \cdot \sin \beta_{5}-T_{6} \cdot \sin \beta_{6}-N_{7} \cdot \sin \alpha_{7} \cdot \cos \beta_{7} \cdot\left(x+l_{H 4}\right)-Q_{n 7} \cdot \sin \beta_{7} \cdot\left(x+l_{H 4}\right)+Q_{\tau 7} \cdot \cos \alpha_{7} \cdot$ $\cos \beta_{7} \cdot\left(x+l_{H 4}\right)-N_{8} \cdot \sin \alpha_{8} \cdot \cos \beta_{8} \cdot\left(x+l_{H 4}\right)-Q_{n 8} \cdot \sin \beta_{8} \cdot\left(x+l_{H 4}\right)+Q_{\tau 8} \cdot \cos \alpha_{8} \cdot \cos \beta_{8} \cdot\left(x+l_{H 4}\right)-M_{\tau 7} \cdot$ $\cos \beta_{7}+M_{\tau 8} \cdot \cos \beta_{8}+T_{7} \cdot \sin \beta_{7}-T_{8} \cdot \sin \beta_{8}-N_{9} \cdot \sin \alpha_{9} \cdot \cos \beta_{9} \cdot x-Q_{n 9} \cdot \sin \beta_{9} \cdot x+Q_{\tau 9} \cdot \cos \alpha_{9} \cdot \cos \beta_{9} \cdot x-N_{10}$. $\sin \alpha_{10} \cdot \cos \beta_{10} \cdot x-Q_{n 10} \cdot \sin \beta_{10} \cdot x+Q_{\tau 10} \cdot \cos \alpha_{10} \cdot \cos \beta_{10} \cdot x-M_{\tau 9} \cdot \cos \beta_{9}+M_{\tau 10} \cdot \cos \beta_{10}+T_{9} \cdot \sin \beta_{9}-T_{10}$. $\left.\sin \beta_{10}\right)^{2} d x$;

$U_{\text {Bend }}\left(l_{H 6}\right)=\frac{1}{2 E I} \int_{0}^{l_{H 6}}\left(M_{G}+G \cdot\left(x+l_{H 1}+l_{H 2}+l_{H 3}+l_{H 4}+l_{H 5}\right)+q \frac{\left(x+l_{H 1}+l_{H 2}+l_{H 3}+l_{H 4}+l_{H 5}\right)^{2}}{2}-N_{1} \cdot \cos \beta_{1} \cdot\right.$ $\left(x+l_{H 1}+l_{H 2}+l_{H 3}+l_{H 4}+l_{H 5}\right)-Q_{n 1} \cdot \sin \beta_{1} \cdot\left(x+l_{H 1}+l_{H 2}+l_{H 3}+l_{H 4}+l_{H 5}\right)-N_{3} \cdot \sin \alpha_{3} \cdot \cos \beta_{3} \cdot$ $\left(x+l_{H 2}+l_{H 3}+l_{H 4}+l_{H 5}\right)-Q_{n 3} \cdot \sin \beta_{3} \cdot\left(x+l_{H 2}+l_{H 3}+l_{H 4}+l_{H 5}\right)+Q_{\tau 3} \cdot \cos \alpha_{3} \cdot \cos \beta_{3} \cdot\left(x+l_{H 2}+l_{H 3}+l_{H 4}+\right.$ $\left.l_{H 5}\right)-N_{4} \cdot \sin \alpha_{4} \cdot \cos \beta_{4} \cdot\left(x+l_{H 2}+l_{H 3}+l_{H 4}+l_{H 5}\right)-Q_{n 4} \cdot \sin \beta_{4} \cdot\left(x+l_{H 2}+l_{H 3}+l_{H 4}+l_{H 5}\right)+Q_{\tau 4} \cdot \cos \alpha_{4} \cdot \cos \beta_{4} \cdot$ $\left(x+l_{H 2}+l_{H 3}+l_{H 4}+l_{H 5}\right)-M_{\tau 3} \cdot \cos \beta_{3}+M_{\tau 4} \cdot \cos \beta_{4}+T_{3} \cdot \sin \beta_{3}-T_{4} \cdot \sin \beta_{4}-N_{5} \cdot \sin \alpha_{5} \cdot \cos \beta_{5} \cdot$

$\left(x+l_{H 3}+l_{H 4}+l_{H 5}\right)-Q_{n 5} \cdot \sin \beta_{5} \cdot\left(x+l_{H 3}+l_{H 4}+l_{H 5}\right)+Q_{\tau 5} \cdot \cos \alpha_{5} \cdot \cos \beta_{5} \cdot\left(x+l_{H 3}+l_{H 4}+l_{H 5}\right)-N_{6} \cdot \sin \alpha_{6} \cdot$ $\cos \beta_{6} \cdot\left(x+l_{H 3}+l_{H 4}+l_{H 5}\right)-Q_{n 6} \cdot \sin \beta_{6} \cdot\left(x+l_{H 3}+l_{H 4}+l_{H 5}\right)+Q_{\tau 6} \cdot \cos \alpha_{6} \cdot \cos \beta_{6} \cdot\left(x+l_{H 3}+l_{H 4}+l_{H 5}\right)-M_{\tau 5}$. $\cos \beta_{5}+M_{\tau 6} \cdot \cos \beta_{6}+T_{5} \cdot \sin \beta_{5}-T_{6} \cdot \sin \beta_{6}-N_{7} \cdot \sin \alpha_{7} \cdot \cos \beta_{7} \cdot\left(x+l_{H 4}+l_{H 5}\right)-Q_{n 7} \cdot \sin \beta_{7} \cdot\left(x+l_{H 4}+l_{H 5}\right)+$ $Q_{\tau 7} \cdot \cos \alpha_{7} \cdot \cos \beta_{7} \cdot\left(x+l_{H 4}+l_{H 5}\right)-N_{8} \cdot \sin \alpha_{8} \cdot \cos \beta_{8} \cdot\left(x+l_{H 4}+l_{H 5}\right)-Q_{n 8} \cdot \sin \beta_{8} \cdot\left(x+l_{H 4}+l_{H 5}\right)+Q_{\tau 8} \cdot$ $\cos \alpha_{8} \cdot \cos \beta_{8} \cdot\left(x+l_{H 4}+l_{H 5}\right)-M_{\tau 7} \cdot \cos \beta_{7}+M_{\tau 8} \cdot \cos \beta_{8}+T_{7} \cdot \sin \beta_{7}-T_{8} \cdot \sin \beta_{8}-N_{9} \cdot \sin \alpha_{9} \cdot \cos \beta_{9} \cdot\left(x+l_{H 5}\right)-$ $Q_{n 9} \cdot \sin \beta_{9} \cdot\left(x+l_{H 5}\right)+Q_{\tau 9} \cdot \cos \alpha_{9} \cdot \cos \beta_{9} \cdot\left(x+l_{H 5}\right)-N_{10} \cdot \sin \alpha_{10} \cdot \cos \beta_{10} \cdot\left(x+l_{H 5}\right)-Q_{n 10} \cdot \sin \beta_{10} \cdot\left(x+l_{H 5}\right)+$ $Q_{\tau 10} \cdot \cos \alpha_{10} \cdot \cos \beta_{10} \cdot\left(x+l_{H 5}\right)-M_{\tau 9} \cdot \cos \beta_{9}+M_{\tau 10} \cdot \cos \beta_{10}+T_{9} \cdot \sin \beta_{9}-T_{10} \cdot \sin \beta_{10}-N_{11} \cdot \sin \alpha_{11} \cdot \cos \beta_{11} \cdot x-$ $\left.Q_{n 11} \cdot \sin \beta_{11} \cdot x+Q_{\tau 11} \cdot \cos \alpha_{11} \cdot \cos \beta_{11} \cdot x-M_{\tau 11} \cdot \cos \beta_{11}+T_{11} \cdot \sin \beta_{11}\right)^{2} d x$. 
The function of potential energy of deformation of the torque moments:

$U_{\text {Tor }}=\frac{1}{2 G J_{p}} \cdot\left[\int_{0}^{l_{H 1}}\left[T\left(l_{H 1}\right)\right]^{2} d x+\int_{0}^{l_{H 2}}\left[T\left(l_{H 1}\right)+T\left(l_{H 2}\right)\right]^{2} d x+\int_{0}^{l_{H 3}}\left[T\left(l_{H 1}\right)+T\left(l_{H 2}\right)+T\left(l_{H 3}\right)\right]^{2} d x+\right.$

$\int_{0}^{l_{H 4}}\left[T\left(l_{H 1}\right)+T\left(l_{H 2}\right)+T\left(l_{H 3}\right)+T\left(l_{H 4}\right)\right]^{2} d x+\int_{0}^{l_{H 5}}\left[T\left(l_{H 1}\right)+T\left(l_{H 2}\right)+T\left(l_{H 3}\right)+T\left(l_{H 4}\right)+T\left(l_{H 5}\right)\right]^{2} d x+$

$\left.\int_{0}^{l_{H 6}}\left[T\left(l_{H 1}\right)+T\left(l_{H 2}\right)+T\left(l_{H 3}\right)+T\left(l_{H 4}\right)+T\left(l_{H 5}\right)+T\left(l_{H 6}\right)\right]^{2} d x\right]$,

where

$T\left(l_{H 1}\right)=N_{1} \cdot \cos \beta_{1} \cdot \sin \alpha_{1} \cdot \frac{b}{2}+Q_{n 1} \cdot \sin \beta_{1} \cdot \sin \alpha_{1} \cdot \frac{b}{2}-Q_{t 1} \cdot \cos \beta_{1} \cdot \cos \alpha_{1} \cdot \frac{b}{2}-M_{n 1} \cdot \sin \alpha_{1}-T_{1} \cdot \cos \alpha_{1}-q \frac{b^{2}}{2} ;$

$T\left(l_{H 2}\right)=N_{3} \cdot \cos \beta_{3} \cdot \sin \alpha_{3} \cdot \frac{b}{2}+Q_{n 3} \cdot \sin \beta_{3} \cdot \sin \alpha_{3} \cdot \frac{b}{2}-Q_{t 3} \cdot \cos \beta_{3} \cdot \cos \alpha_{3} \cdot \frac{b}{2}-M_{n 3} \cdot \sin \alpha_{3}-T_{3} \cdot \cos \alpha_{3}+N_{4}$.

$\cos \beta_{4} \cdot \sin \alpha_{4} \cdot \frac{b}{2}+Q_{n 4} \cdot \sin \beta_{4} \cdot \sin \alpha_{4} \cdot \frac{b}{2}-Q_{t 4} \cdot \cos \beta_{4} \cdot \cos \alpha_{4} \cdot \frac{b}{2}-M_{n 4} \cdot \sin \alpha_{4}-T_{4} \cdot \cos \alpha_{4}-q \frac{b^{2}}{2} ;$

$T\left(l_{H 3}\right)=N_{5} \cdot \cos \beta_{5} \cdot \sin \alpha_{5} \cdot \frac{b}{2}+Q_{n 5} \cdot \sin \beta_{5} \cdot \sin \alpha_{5} \cdot \frac{b}{2}-Q_{t 5} \cdot \cos \beta_{5} \cdot \cos \alpha_{5} \cdot \frac{b}{2}-M_{n 5} \cdot \sin \alpha_{5}-T_{5} \cdot \cos \alpha_{5}+N_{6}$.

$\cos \beta_{6} \cdot \sin \alpha_{6} \cdot \frac{b}{2}+Q_{n 6} \cdot \sin \beta_{6} \cdot \sin \alpha_{6} \cdot \frac{b}{2}-Q_{t 6} \cdot \cos \beta_{6} \cdot \cos \alpha_{6} \cdot \frac{b}{2}-M_{n 6} \cdot \sin \alpha_{6}-T_{6} \cdot \cos \alpha_{6}-q \frac{b^{2}}{2} ;$

$T\left(l_{H 4}\right)=N_{7} \cdot \cos \beta_{7} \cdot \sin \alpha_{7} \cdot \frac{b}{2}+Q_{n 7} \cdot \sin \beta_{7} \cdot \sin \alpha_{7} \cdot \frac{b}{2}-Q_{t 7} \cdot \cos \beta_{7} \cdot \cos \alpha_{7} \cdot \frac{b}{2}-M_{n 7} \cdot \sin \alpha_{7}-T_{7} \cdot \cos \alpha_{7}+N_{8} \cdot$

$\cos \beta_{8} \cdot \sin \alpha_{8} \cdot \frac{b}{2}+Q_{n 8} \cdot \sin \beta_{8} \cdot \sin \alpha_{8} \cdot \frac{b}{2}-Q_{t 8} \cdot \cos \beta_{8} \cdot \cos \alpha_{8} \cdot \frac{b}{2}-M_{n 8} \cdot \sin \alpha_{8}-T_{8} \cdot \cos \alpha_{8}-q \frac{b^{2}}{2} ;$

$T\left(l_{H 5}\right)=N_{9} \cdot \cos \beta_{9} \cdot \sin \alpha_{9} \cdot \frac{b}{2}+Q_{n 9} \cdot \sin \beta_{9} \cdot \sin \alpha_{9} \cdot \frac{b}{2}-Q_{t 9} \cdot \cos \beta_{9} \cdot \cos \alpha_{9} \cdot \frac{b}{2}-M_{n 9} \cdot \sin \alpha_{9}-T_{9} \cdot \cos \alpha_{9}+N_{10}$.

$\cos \beta_{10} \cdot \sin \alpha_{10} \cdot \frac{b}{2}+Q_{n 10} \cdot \sin \beta_{10} \cdot \sin \alpha_{10} \cdot \frac{b}{2}-Q_{t 10} \cdot \cos \beta_{10} \cdot \cos \alpha_{10} \cdot \frac{b}{2}-M_{n 10} \cdot \sin \alpha_{10}-T_{10} \cdot \cos \alpha_{10}-q \frac{b^{2}}{2}$;

$T\left(l_{H 6}\right)=N_{11} \cdot \cos \beta_{11} \cdot \sin \alpha_{11} \cdot \frac{b}{2}+Q_{n 11} \cdot \sin \beta_{11} \cdot \sin \alpha_{11} \cdot \frac{b}{2}-Q_{t 11} \cdot \cos \beta_{11} \cdot \cos \alpha_{11} \cdot \frac{b}{2}-M_{n 11} \cdot \sin \alpha_{11}-T_{11} \cdot$

$\cos \alpha_{11}-q \frac{b^{2}}{2}$.

The full expression of the function of potential energy of deformation is extremely long though it is not too complicated in its recording structure as it involves some gradual addition of the function constituents on the sections series of the structure. The unknown support reactions $R_{l}$, $R_{2}, R_{3}$ and $R_{4}$ are found from the static equations

$\sum F(x)=0:-R_{1}-R_{3}=0$;

$\sum F(z)=0: R_{2}+R_{4}-G-q \frac{1}{2} \sum_{i=1}^{6} l_{H}=0 ;$

$\sum M(0)=0: M_{G}+G \cdot \sum_{i=1}^{6} l_{H}+q \cdot \frac{1}{2}\left(\sum_{i=1}^{6} l_{H}\right)^{2}-R_{4} \cdot$ $L_{R_{4}}-R_{3} \cdot L_{R_{3}}=0$.

And some extra equation, obtained from the condition (3) by the differentiation of expressions (4) and (5) by the unknown $R_{I}$ followed by the integration (the equation can't be written due to its awkwardness).

The peculiarity of solving the problems of abovementioned type is the fact that whilst writing the expression of the potential energy of deformation it's necessary to express the required unknown internal efforts in terms of those unknown ones which the partial derivatives are taken by. Thus, to solve the equations it's quite reasonable to use
Leibniz rule of the integrand (function under the integral sign) differentiation (i.e. at first the partial derivatives must be taken and only after that the integration can be performed) or other approaches $[16,17]$ which make the calculations faster and simpler. To analyze the distribution of efforts in the bearing structures of the machine it's quite reasonable to use some computational investigation based on the simulation models [18-21].

\section{Results and discussion}

The output data for the crop sprayer boom section calculation are its geometrical parameters, design and processing mass. The length of the crop sprayer boom $L=$ $13 \mathrm{~m}$. The design mass of the boom $m_{K}=238 \mathrm{~kg}$. The weight of processing equipment mounted on the boom (hydraulic cylinders, compensating springs, pipelines, hoses, nozzle valves and others) is equal to $m_{T} \approx 160 \mathrm{~kg}$.

Uniformly distributed load on the boom section will be the following one:

$q=\frac{\left(m_{K}+m_{T}\right) g}{L}=(238+160) \cdot \frac{9.81}{13} \approx 300 \mathrm{~N} / \mathrm{m}$. 
The length of two end boom sections $L_{S} \approx 7 \mathrm{~m}$, design mass $m_{S}=88 \mathrm{~kg}$. We have simulated the impact of two end boom sections on the first section by the external effort $G$ and the moment $M_{G}$ :

$G=q \cdot L=300 \cdot 7=2100 \mathrm{~N} ;$

$M_{G}=G \cdot L / 2=2100 \cdot 7 / 2=7350 \mathrm{~N} \cdot \mathrm{m}$.

The results of analytical calculation have been checked by the simulation of 3D model of the crop spraying machine boom section (Fig. 4) using the program complex «LiraSAPR», where the finite elements method (FEM) has been implemented.

To simplify the support reactions determination some additional pin-jointed components have been introduced into the crop sprayer boom section structure. They imitate horizontal and vertical constituents of the support reactions.
The calculation scheme of the boom section under discussion and the results of checking are shown on the Figure 5.

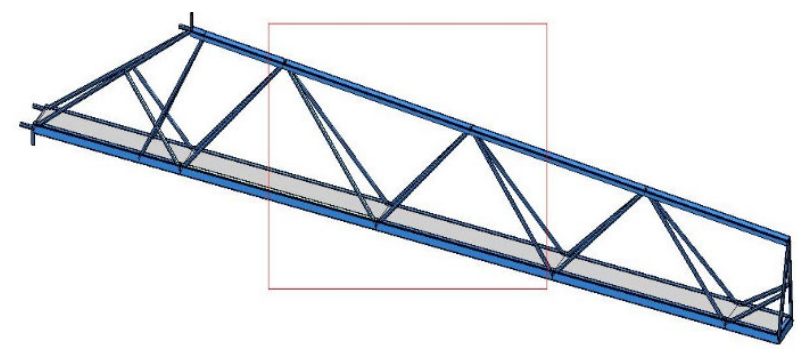

Fig. 4. 3D model of the crop spraying machine boom section

The comparison of results of analytical and simulation modeling is shown in Table 1.

a)

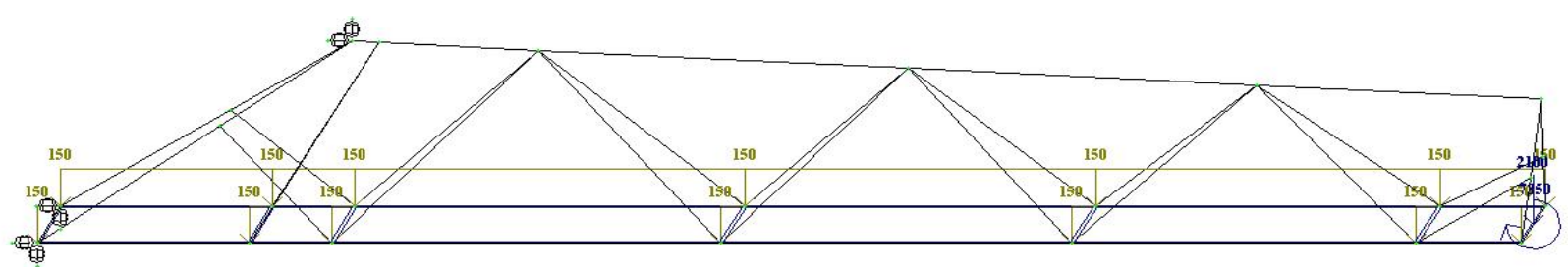

b)

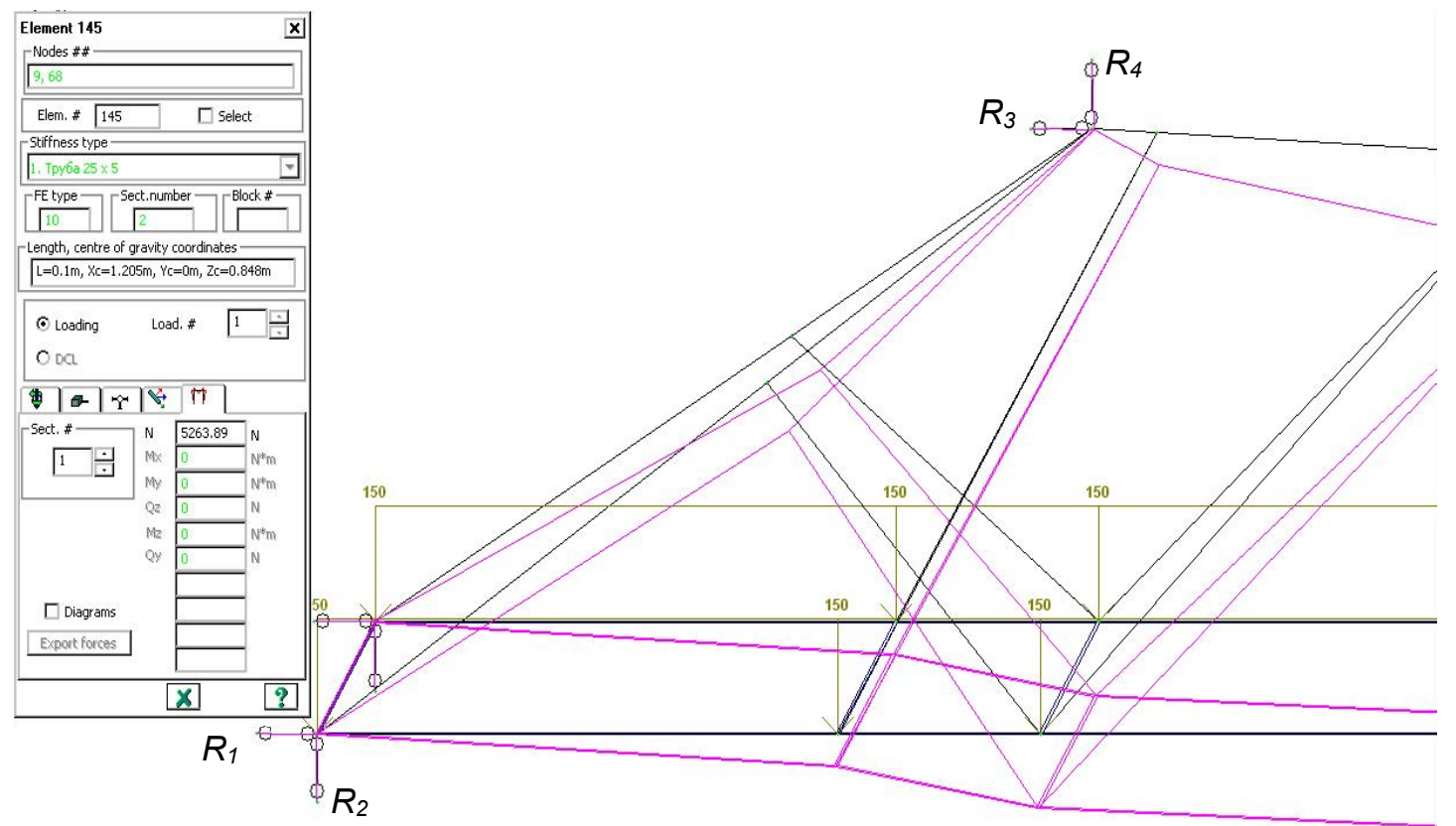

Fig. 5. Modeling of the crop spraying boom section: a) scheme of the structure fixing and loading; b) results of calculation (scheme of deformation and support reaction $\mathrm{R}_{4}$ ) 
Table 1.

Comparison of the research results

\begin{tabular}{cccc}
\hline $\begin{array}{c}\text { Reactions of } \\
\text { supports }\end{array}$ & $\begin{array}{c}\text { Analytical solution } \\
\text { (MPED method) }\end{array}$ & $\begin{array}{c}\text { Simulation } \\
\text { (FEM) }\end{array}$ & Difference \\
\hline $\mathrm{R}_{1}, \mathrm{H}$ & $-13927 / 2 \approx-6964$ & -7048.32 & $\approx 1 \%$ \\
\hline $\mathrm{R}_{2}, \mathrm{H}$ & $-1512 / 2 \approx-756$ & -697.70 & $\approx 8 \%$ \\
\hline $\mathrm{R}_{3}, \mathrm{H}$ & 13927 & 14096.60 & $\approx 1 \%$ \\
\hline $\mathrm{R}_{4}, \mathrm{H}$ & 5377 & 5263.89 & $\approx 2 \%$ \\
\hline
\end{tabular}

\section{Conclusions}

The results of theoretical investigation of efforts distribution in the supports of the spraying machine boom section in static statement of the problem have been described. The method of static indeterminacy realization based on the principle of minimum of potential energy of deformation has been used to calculate the frame structure.

The applied approach under discussion enables the static indeterminacy to be realized whilst calculating the complex frame structures though it requires not only considerable records of the function of potential energy of deformation (even without taking into account the function of potential energy of deformation of normal and lateral forces) but application of applied computer programs to perform a large volume of differentiation operations and integration of expressions components of the function of potential energy of deformation by unknown efforts as well.

Results of theoretical calculation and simulation of the spraying machine boom section make possible to find any defects in the spraying machine boom section design, determine the most load-carrying structural members and choose the most efficient design parameters.

Difference in the results obtained analytically by method of minimum of potential energy of deformation and by means of simulation does not exceed $10 \%$.

\section{References}

[1] V.I. Kravchuk, D.H. Voitiuk, Machines for chemical plant protection, Doslidnytske, UkrNDIPVT, Ukraine, 2010 (in Ukrainian).

[2] O.S. Baranovskyi, V.V. Marchenko, Mechanical and technological principles of effective use of pesticides in spraying, Ahrarna Tekhnika ta Obladnannya Agricultural Machinery and Equipment 4/5 (2008) 3438 (in Ukrainian).

[3] S. Afanasiev, V. Horbatov, V. Pohorilyi, Qualitative element base - the basis of reliability of domestic equipment, Tekhnika APK - Agroindustrial Complex Technical 5/6 (2006) 40-43 (in Ukrainian).

[4] M.F. Dmytrychenko, I.A. Vikovych, Dynamics of mobile machines with hinged functional elements, LPI Publ., Lviv, Ukraine, 2008 (in Ukrainian).

[5] T.I. Rybak, Search design based on resource optimization of mobile agricultural machines, TVPK Zbruch, Ukraine, 2003 (in Ukrainian).

[6] P.M. Volkov, M.M. Fundamentals of the theory and calculation of agricultural machinery for strength and reliability, Mashinostroyeniye, Moscow, USSR, 1977 (in Russian).

[7] N.K. Ashirbayev, Zh.N. Ashirbayeva, Sh.E. Altynbekov, M.T. Shomanbayeva, Features distribution of dynamic perturbations in an elastic multisupport construction, Bulletin of the Karaganda University - Mathematics 2/82 (2016) 8-14. DOI: https://doi.org/10.1063/1.4959653

[8] G.A. Yessenbayeva, K.S. Kutimov, Zh.R. Sazhinova, A.Zh. Sarsenbek, On the application of mathematical methods for the research of vibration processes in mechanics, Bulletin of the Karaganda University Mathematics 2/86 (2017) 55-63.

DOI: https://doi.org/10.31489/2017m2/55-63

[9] E. Arinov, S.Zh. Karipbaev, K.Z. Sartayev, Dynamic stress-strain state of a single-section manipulator, Bulletin of the Karaganda University - Mathematics 3/87 (2017) 27-32.

DOI: https://doi.org/10.31489/2017m3/27-32

[10] R.B. Hevko, I.G. Tkachenko, S.V. Synii, I.V. Flonts, Development of design and investigation of operation processes of small-sclale root crop and potato harvesters, INMATEH: Agricultural Engineering 49/2 (2016) 53-60.

[11] H.S. Pysarenko, Strength of Materials, Viyshcha Shkola, Kyiv, 1974 (in Ukrainian).

[12] T. Rybak, M. Stashkiv, Machines loadbearing structures thin-walled elements features of calculation, Visnyk TDTU - Bulletin Ternopil Ivan Puluj National Technical University 8/2 (2008) 34-39 (in Ukrainian). 
[13] T.I. Rybak, M.Y. Stashkiv, Y.Y. Ripetskyy, Application of the modified method of a minimum of potential energy of deformation at calculation of loadbearing systems of agricultural machines in dynamic statement of a problem, Visnyk Kharkivskoho Natsionalnoho Tekhnichnoho Universytetu Silskoho Hospodarstva imeni Petra Vasylenka - Bulletin of the Kharkiv National Technical University of Agriculture 69 (2008) 80-84 (in Ukrainian).

[14] P.V. Popovych, M.Y. Stashkiv, H.B. Dutka, T.A. Dovbush, Energy method for detecting static uncertainty of loadbearing frames of mobile agricultural machines, Visnyk Kharkivskoho Natsionalnoho Tekhnichnoho Universytetu Silskoho Hospodarstva im. Petra Vasylenka - Bulletin of the Kharkiv National Technical University of Agriculture 120 (2012) 220-225 (in Ukrainian).

[15] M. Pidhurskyy, M. Stashkiv, I. Pidhurskyy, Features damage propagation in statically indeterminate rod systems analysis, Visnyk TNTU - Bulletin Ternopil Ivan Puluj National Technical University 2 (2011) 118123 (in Ukrainian).

[16] P. Popovych, L. Poberezhny, O. Shevchuk, Evaluation of strength of carrying metal structures of trailers, Journal of Achievements in Materials and Manufacturing Engineering 100/2 (2020) 58-69. DOI: https://doi.org/10.5604/01.3001.0014.3345

[17] B.T. Kalimbetov, Kh.F. Etmishev, Asymptotic solutions of scalar integro-differential equations with partial derivatives and with rapidly oscillating coefficients, Bulletin of the Karaganda University Mathematics 1/97 (2020) 52-67.

DOI: https://doi.org/10.31489/2020m1/52-67

[18] M. Bukenov, A. Ibrayev, D. Zhussupova, D. Azimova, Numerical solution of a problem on bending oscillation of a rod, Bulletin of the Karaganda University Mathematics 2/86 (2017) 32-36.

DOI: https://doi.org/10.31489/2017m2/32-36

[19] R.B. Hevko, I.G. Tkachenko, Y.B. Hlado, Mathematical model of a root harvester after-cleaning system, Bulletin of the Karaganda university University - Mathematics 4/96 (2019) 81-89. DOI: https://doi.org/10.31489/2019m4/81-89

[20] A. Nanka, I. Morozov, V. Morozov, Improving the efficiency of a sowing technology based on the improved structural parameters for colters, EasternEuropean Journal of Enterprise Technologies 4/1(100) (2019) 33-45. DOI: https://doi.org/10.15587/1729$\underline{4061.2019 .174445}$

[21] O. Lyashuk, A. Gupka, Y. Pyndus, V. Gupka, M. Sipravska, A. Wozniak, M. Stashkiv, The tribology of the car: Research methodology and evaluation criteria, Proceedings of the $1^{\text {st }}$ International Scientific Conference "Current Problems of Transport: ICCPT 2019”, Ternopil, 2019, 231-237. DOI: https://doi.org/10.5281/zenodo.3387621
Creative Common International 4.0 Derivat (C) (1) $\Theta \Theta$

(C) 2021 by the authors. Licensee International OCSCO World Press, Gliwice, Poland. This paper is an open access paper distributed under the terms and conditions of the Creative Commons AttributionNonCommercial-NoDerivatives 4.0 International (CC BY-NC-ND 4.0) license (https://creativecommons.org/licenses/by-nc-nd/4.0/deed.en). 